\title{
The Application of Kinesthetic Perception Experience and Environmental Design
}

\author{
Jun Yi \\ Wenhua College \\ Wuhan, China
}

\begin{abstract}
From the perspective of environmental psychology, this article takes a variety of perceptual experience theories as its research background. It elaborates on the meaning of kinesthetic perception, explains the performance level of dynamic perception experience design in depth, and meticulously analyzes the dynamic perception experience in the combination of order and mood and environmental design. From the design perspective of the dynamic perception experience, it tries to provide a variety of different ideas for the environmental design program. It aims to improve the cognitive ability of the public's perception of moving design, deepen the emotion and memory of environmental awareness, and enrich the space and form of environmental design works.
\end{abstract}

Keywords-kinesthetic perception; experience design; emotional design; combination mode

\section{INTRODUCTION}

For the concept of environmental perception, the definitions are different. Psychologists emphasize the meaning of neurophysiology and psychology. The anthropologists regard "perception" as a cultural experience related to emotional reactions, values, and cultural heritage. The phenomenological philosophers believe that "perception" is the starting point for people to understand things. And it is the perception of experience. It can only be described by the method. We cannot use "objective thinking" to explain it. The planners and design staff prefer the general "feelings" and "experience" to cover "perception" and "cognition". It includes a wider range than psychology [1]. Whatever the definition, it is undeniable that environmental perception is important to the human experience and cognitive environment.

The environment can provide information for humans through various sensory perceptions, mainly including five perceptual cognitions: visual perception, auditory perception, olfactory perception, tactile perception, and kinesthetic perception. Vision is one of the most acceptable and simple sensory channels. It is widely used and designed. As an auxiliary means of visual experience, the position of hearing is second to the position of vision. Therefore, the design of the scene is everywhere. The sense of smell can make people have different memory of place or city through various kinds of odors, and enhance the perception. The touch creates a touchable world, which is an important means for human beings to understand things deeply. The cognitive experience of kinesthesia is related to people's body, vision and emotion Compared with the other four senses, kinesthesia is more complex and deep-seated cognitive experience way. Researching the design of dynamic perception experience not only needs the help of information from other sensory perception experiences, but also has certain requirements on the sensitivity and observation of human beings. And even, it requires that the information receiver has a higher ability to understand the conception and strong thinking logic ability. The connotation of the design works could be truly transmitted to human beings. Human can perceive the characteristics and situations of things more comprehensively and more abundantly.

\section{The MeAning OF KInESTHETIC PERCEPTION}

\section{A. The Concept of Kinesthetic Perception}

Kinaesthetic perception is the feeling of body movement and its positional state. It is related to muscle tissue, tendon and joint activities [1]. When humans move, they change their position, the shift of vision and the change of mood, such as walking, running, climbing or jumping, will make people have different viewing experience and emotion cognition. And the speed of walking and the direction of running will be different. The high and low change of climbing or the rhythm of jumping will promote different degree of emotion resonance and environment cognition.

\section{B. Analysis on the Relationship between Kinesthetic Perception and Environmental Design}

The relationship between kinesthetic perception and environmental design is mutually reinforcing and extremely close. Good design of kinesthetic experience can help human to obtain environmental information sufficiently and make up for the deficiency of other perceptual cognition. On the contrary, the function and nature of environment design decides the design form of motion perception. Therefore, reasonable understanding and use of the relationship between kinesthetic perception and environmental design is conducive to the effectiveness of the human cognitive environment and the interest of environmental design.

\section{Significance of Kinesthetic Perceptual Experience}

Kinetic perception is one of the important ways for human to experience the environment. The special 
kinesthetic experience design is helpful to the formation of the characteristic landscape, and the generation of the unique emotional memory. To a certain extent, it also can make the person produce the synesthesia, stimulate the other perceptual common function, and promote the development of the various perceptual patterns. For example, in the process of climbing a mountain, the constant movement of the body creates a sense of fatigue. And then, there must have a pause at some point in the rest. The position of the body, the direction of the sight and the fluctuation of the mood are all changing at this time. And then, people will be attracted by the strong visual scenery on the mountain. Some kind of floral fruity fragrance would attract them. The kinesthetic behavior will be combined with the visual cognition. This kind of sudden combination has brought the infinite surprise and the expectation to the person in the journey.

\section{THE HIERARCHICAL RELATIONSHIP OF KINESTHETIC PERCEPTION EXPERIENCE DESIGN}

The form of kinesthetic experience design is produced by the movement of human body position, the shift of sight and the stimulation of different emotions. The three levels of presentation gradually deepen the cognitive level of kinesthetic experience.

\section{A. Movement of Body Position}

The movement of body position is the most basic expression of kinesthetic experience. The movement of position, direction, speed and other factors will affect the acquisition of environmental cognitive information. The movement of body position can be divided into active movement and passive movement.

Active movement is a kind of spontaneous behavior movement produced by human beings according to selfconsciousness. And it has the characteristics of autonomy and arbitrariness. In the design of the park, square and street in the external environment, the designer will plan the route of the good man and the car, the location of each landscape node, the connection among the nodes, and even the visitor's tour speed control and so on. However, the receiver does not necessarily act according to the intrinsic line. They may make some common behavior in the outer space, such as cutting a corner. Humans cannot experience kinesthesia on the basis of a designed circuit. To some extent, they will miss some arranged landscape nodes. However, it is also possible to produce different kinesthetic experience.

Passive movement is a kind of compulsory behavior movement produced by human beings according to the planned line, which has the characteristics of being forced and following. In the design of exhibition spaces such as exhibition halls and museums, the main reason is that visitors can enjoy all the works in an orderly and complete manner according to the route planned by the designer. For example, the design of the visiting route of IKEA home can effectively guide and divide the flow of people through the "one-line" route. The customers can feel each type of products of IKEA in the constant movement of location. It virtually prolongs the process of shopping time and experience. Also, it promotes consumption. And it can force customers to feel the culture of the enterprise. Of course, regular customers will find that IKEA has short-cut road signs. According to their own circumstances, the customers could choose the demand. And then, it would avoid having the sense of boredom and fatigue brought by long-time shopping.

\section{B. Diversion of the Sight}

The shift of the sight is the second level of kinesthetic experience. When the movement of the body pauses, the shift of the sight will occur. The landscape of the transfer point is the unexpected surprise in kinesthetic experience.

For example, the traditional Chinese garden in the planning will use the design method of "walking landscape difference". In the process of movement, the position of the body changes, and the direction of the sight would change. People would have a wealth of landscape experience. However, all "scenery" in the planning and design are reasonable arrangements. Their settings and design content are related to the experience of the body in the movement. That is to say, "scenic spots" must have reasonable logic and order. The climbing corridor in the Summer Palace is to take advantage of the changes in the topographic difference. Along the mountain corridor, it has unique scenery. At each corner, people can meet unique landscape. From the bottom of the mountain to the hillside and then to the top of the mountain, from looking up to looking down, we can see the Buddha statues from different angles. They would have unique feelings of kinesthetic experience.

\section{Stimulation of Different Emotions}

The stimulation of different emotions is the third level of kinesthetic experience. When both the body and the eye are moving, one's emotions will be constantly stimulated. They may be excited, anxious, confused, or cheerful.

For example, in a labyrinth garden, a child is willing to keep looking for a way out. With the change of body position, the sight and brain thinking, he will even produce high-pitched emotions. The interesting environment design just stimulates his curiosity. He will be very happy to enjoy the process of exploration and kinesthetic experience. However, the old man walks, and he cannot find the outlet. He would produce the anxious and panic mood because of the tired body. The uncertain environment design has stimulated his uneasy psychology. And he will not be willing to accept this process of dull movement experience. And then, people in different ages and different psychological bearing stage, would encounter the same kinesthetic environment experience. However, it will produce different emotional experience.

\section{The Combination Mode OF Kinesthetic PERCEPTUAL EXPERIENCE AND ENVIRONMENTAL DESIGN}

The application of kinesthetic perceptual experience in environmental design is very extensive. And the main basis of its combination is the three levels of kinesthetic expression: kinesthetic line, order and emotional design. 


\section{A. Design of Kinesthetic Line}

In the environment design, the movement of the body position is the direct embodiment of the kinesthetic line design. The kinesthetic line is a line strung together by the points that a person moves in the environment. This line can guide people's behavior and consciousness. It is a very important element in environmental design. Different kinesthetic line design will produce different changes in body position. People would see different landscape. Also, people would have different emotions. However, what is good design of kinesthetic line? The premise is that it must conform to the function, nature and culture of the design of the environment. And it must be consistent with the human psychology.

Kinesthetic line can be functional or formal. In different environment, the same kinesthetic line design would produce different effects. For example, accessibility and convenience is the primary principle of kinesthetic line design. However, due to people, time, place and cultural differences, it would have different choices. When waiting in line in front of a pavilion of Shanghai World Expo, in order to avoid crowd congestion, emotional anxiety and confusion of the site, the U-shaped complex line is used in the design of the kinesthetic line. People can move as far as possible. And it would reduce physical friction. At the same time, it would alleviate the uneasy psychology. In the planning of the kinesthetic line of traditional Chinese garden, in order to make the limited area show infinite sense of space, the design of traditional Chinese garden will be prolonged and complicated. With the changes of the body movement, people will ignore the small scale of the garden. They will focus on the way to appreciate the landscape and aesthetic experience of the garden.

Also, we should take the passive mobile line to display space kinesthetic line design as far as possible. The visitors would have kinesthetic experience in accordance with the design ideas. However, different design will produce active mobile experience effect. The design of the rockery stone forest in the Suzhou Lion Forest is very interesting. It is divided into three levels: upper, middle, and lower. There are 9 mountain roads and 21 caverns. The visitors should follow the winding path. Sometimes, they should go through the hole and walk across the bridge. It is so interesting. There are stone peaks and stalagmites on the rockery. There are various shapes and postures. It was said that when the emperor visited this rockery, he had been walking in the rocks for a long time and could not get out of it. Each time he moved his body, he saw different scene, which made him happy. He believed that this experience was "interesting". Thus, it can be seen that the seemingly passive kinesthetic line design can also successfully attract people's active exploration, stimulate people's visual and tactile feelings, form a variety of perceptual patterns, and complete the comfortable kinesthetic experience process.

\section{B. Order Design}

The direct manifestation of the shift of the sight in environmental design is the order design. The synonyms of order are "system", "structure" and "mode". It is a difficult concept to grasp [2]. There is some interpretation related to the order: sequence, organization, the law and so on. The simple understanding of order design is to make the design has a certain logic, level and order. And then, people wouldn't have fuzzy, messy or elusive feelings.

For example, total length of the long corridor design of the Summer Palace is 728 meters. There are a total of 273 rooms. From the perspective of environmental psychology, the body will certainly produce a sense of exhaustion in the tour. However, the design of the Long Corridor of the Summer Palace fully considers the physical and psychological needs of people. With rigorous logic and order, it plans out the Liujia Pavilion, Jilan Pavilion, Qiushui Pavilion and Qingyao Pavilion from east to west, representing the four seasons of spring, summer, autumn and winter respectively. In addition, there are more than 14000 pictures on each of the fangliang of the corridor, including landscapes, flowers, birds, fish and insects, and people's stories, etc. With the orderly arrangement of the direction of sight, the kinesthetic experience can be carried out effectively and easily. And then, it has formed perfect and comfortable process of experience.

\section{Emotional Design}

The direct expression of the stimulation of different emotional in environmental design is emotional design. Emotion is the possibility and inevitability of the body's success in behavior. The evaluation and experience of physical response includes joy, anger, sadness, shock, fear, love and so on. From a psychological point of view, emotion is produced by the influence of external environment. Such emotions are usually characterized by subjectivity, contingency and one-sidedness.

Chinese traditional garden is good at using the creative means of "promoting the first suppression". It would promote the change of human emotion through the size of space. Then, it would make changes from compact to loose, from repression to comfortable, and from the beginning to the climax.

The entrance design of Suzhou Garden is a good example. A huge garden has small courtyard and narrow entrance passage. Firstly, it would give people depressed and impetuous feelings. This narrow entrance passage is more than 50 meters long. And it uses the way of making scenery by blocking and borrowing scenery. With the contrast of the size, the brightness and darkness, it has formed rich and changeable space. It makes the narrow passages that are full of life. Once people walk through this passage, they will be able to see the main landscape in the garden. They would see the landscape stone scenery at a glance. And they would forget the original oppressed mood. People would make the design and interpretation of space with emotional change in the kinesthetic experience. People could have emotional sublimation in kinesthetic experience.

\section{CONCLUSION}

It is not difficult to find that the environment design can be well combined with kinesthetic perceptual experience 
through the discussion of the full text and the exploration of the combination mode. The significance of the combination is that we can feel the different changes of body movement and mood fluctuation, and change our static life state through the design of kinesthetic perception. And then, people could have more perceptual experience way, and enjoy the different happiness brought by the kinesthetic. People can design more interesting environmental design works, and enrich our life experience. The style of environmental design would be more diverse. And the experience of the city would be more unique.

\section{REFERENCES}

[1] Hu Zhengfan, Lin Yulian. Environmental psychology [M]. China Architecture \& Building Press, 2013

[2] Hayek. Fatal conceit [M]. Chinese Social Science Press, 2000 